\title{
Long non-coding RNA FTX predicts a poor prognosis of human cancers: a meta-analysis
}

\author{
Weiwei Chen ${ }^{1,2}$, Yuting Li ${ }^{1}$, Liliangzi Guo ${ }^{1}$, Chenxing Zhang ${ }^{1}$ and $\left(\mathbb{5}\right.$ Shaohui Tang ${ }^{1}$ \\ ${ }^{1}$ Department of Gastroenterology, The First Affiliated Hospital, Jinan University, Guangzhou, P. R. China; ${ }^{2}$ Department of Gastroenterology, The First People's Hospital of Zunyi, The \\ Third Affiliated Hospital of Zunyi Medical University, Zunyi, P. R. China
}

Correspondence: Shaohui Tang (tangshaohui206@163.com, tangshaohui206@jnu.edu.cn)

\section{OPEN ACCESS}

Received: 18 November 2020 Revised: 21 December 2020 Accepted: 04 January 2021

Accepted Manuscript online: 05 January 2021

Version of Record published: 14 January 2021
Background: Several studies have assessed the relationship between long non-coding RNA five prime to Xist (FTX) expression, clinicopathological features, and survival outcomes in patients with cancer with conflicting results. This meta-analysis synthesized existing data to clarify the association between FTX with cancer prognosis.

Methods: PubMed, Embase, Cochrane library, Web of Science, Chinese CNKI, and the Chinese WanFang databases were used to search for relevant studies. The role of FTX in cancers was evaluated by pooled odds ratios (ORs) and hazard ratios (HRs) with $95 \%$ confidence intervals (Cls).

Results: Eleven studies comprising 1210 participants including colorectal cancer (CRC), hepatocellular carcinoma (HCC), gastric cancer (GC), renal cell carcinoma (RCC), osteosarcoma (OSC), and glioma were enrolled in this analysis. The meta-analysis showed that high FTX expression was significantly associated with several clinicopathological characteristics, including lymph node metastasis in patients with $\mathrm{CRC}, \mathrm{GC}, \mathrm{HCC}$, and RCC, distant metastasis in patients with $\mathrm{CRC}, \mathrm{GC}, \mathrm{HCC}$, and OSC, larger tumor size in patients with CRC, GC, HCC, RCC, and OSC, and subsequently TNM/clinical stage in patients with CRC, GC, $\mathrm{HCC}$, OSC, and glioma. The pooled results from the survival analysis revealed a significant correlation between high FTX expression and shorter OS in patients with HCC, CRC, GC, OSC, and glioma. Further, FTX overexpression could be an independent predictive marker for shorter OS in patients with CRC, HCC, OSC, and glioma.

Conclusions: FTX may be a potential oncogene, with high FTX expression being associated with a poorer prognosis in patients with $\mathrm{CRC}, \mathrm{HCC}$, OSC, and glioma.

\section{Introduction}

The latest cancer statistics indicate that cancer is the leading cause of human death both in the world and in China [1,2], which results in serious economic burden. Modern treatments for cancer including surgery, adjuvant therapy, and supportive therapy have witnessed a dramatic improvement in recent years. However, many patients are at an advanced stage of cancer at the time of diagnosis, which leads to a poor prognosis.

Long non-coding RNAs (lncRNAs) are RNA molecules comprising a transcription length of more than 200 nucleotides and lack of protein-coding capacity [3]. LncRNAs were previously misunderstood as being the noise of genome transcription with no biological function [4]. However, growing evidence has revealed that lncRNAs are involved in a wide range of biological processes, such as chromosome modification, genome modification, transcriptional activation, transcriptional interference, and other processes [5]. Recent studies have shown that lncRNAs are found to be crucial in regulating cellular processes, such as cell cycle, growth, and apoptosis, which ensure homeostasis [6]. A large number of lncRNAs are associated with various types of cancer, which may exhibit tumor-suppressive and promoting (oncogenic) functions, 
and therefore they hold strong potential as biomarkers for the early diagnosis and prognostic prediction of cancers, and thus, also as targets of molecular targeted therapy [7].

$\mathrm{X}$ chromosome inactivation (XCI), the transcriptional silencing of one $\mathrm{X}$ chromosome that takes place in female mammals to compensate for $\mathrm{X}$-linked gene dosage imbalance, is controlled and regulated by a cis-acting region on the $\mathrm{X}$ chromosome termed the X-inactivation center(Xic). Xist is a lncRNA gene that is the master control point in XCI. LncRNA five prime to Xist (FTX) is a highly conserved transcript of 2300 nucleotides encoded by the FTX gene, which is identified as an activator of Xist in mouse embryonic stem cells and a novel non-coding RNA involved in XCI [8]. Increasing studies have shown that FTX is related to the clinicopathological features and prognosis of patients with cancer, including colorectal cancer (CRC) [9], osteosarcoma (OSC) [10], gastric cancer (GC) [11], and renal cell carcinoma (RCC) [12]. However, these studies were limited by sample size and had opposite outcomes. For example, Liu et al. [13] indicated that FTX was significantly down-regulated in hepatocellular carcinoma (HCC) tissues compared with paired adjacent liver tissues, and the high FTX group exhibited better tumor differentiation, intact tumor encapsulation, smaller tumors, and a better overall survival. Jin et al. reported similar results, FTX expression was down-regulated in non-small-cell lung cancer (NSCLC) clinical tissue samples and cell lines, while ectopic expression of FTX inhibited proliferation and metastasis of lung cancer cells in vitro and in vivo [14]. In contrast, Li et al. [15] revealed that compared with paired adjacent liver tissues, HCC tissues had higher FTX expression that was significantly associated with tumor differentiation and metastasis, and predicted poor prognosis. Zhang et al. found that FTX was significantly up-regulated in gliomas, and up-regulation of FTX significantly promoted proliferation and invasion of glioma cells [16]. Therefore, it is necessary to conduct a comprehensive meta-analysis of the existing data to assess the correlation between FTX and clinicopathological features and survival outcomes in patients with cancer.

\section{Materials and methods Search strategy and study selection}

The study protocol was registered with PROSPERO (registration number: CRD42020199238) before the study began. A systematic literature search was conducted in PubMed, Embase, Cochrane library, Web of Science, Chinese CNKI, and the Chinese WanFang databases from their inception through August 1, 2020.The keywords for searches were 'long non-coding RNA FTX' OR 'LncRNA FTX' AND 'cancers' or 'neoplasm' or 'tumors'. The citation lists of relevant studies were also rigorously screened.

Studies that met the following criteria were included in the analysis: (1) the expression of FTX in patients with cancers was examined in tumor tissues and assessed using qRT-PCR; (2) the results provided survival information including overall survival (OS), disease-free survival (DFS), progression-free survival (PFS), and recurrence-free survival (RFS); (3) hazard ratios (HRs) for survival outcomes were provided or could be calculated from survival curves; and (4) the most recent paper was selected in cases of a repeat study.

The exclusion criteria for this study were as follows: (1) laboratory articles, reviews, case reports, letters, editorials, and conference reports; (2) non-human subject studies; and (3) inability to calculate HR based on the data provided.

\section{Data extraction and quality assessment}

The full texts of potentially eligible articles were scrutinized independently by two authors (W.W.C. and Y.T.L.). Disagreements were resolved by consensus. The extracted data included the first author's name, the year of publication, country, number of cases, type of cancer, clinicopathological features, and survival outcome. The Newcastle-Ottawa scale (NOS) criteria [17] were used to assess the quality of the studies. When the NOS score was $\geq 6$, the article was considered to be of high-quality. An NOS score $<6$ was indicative of a low-quality study.

\section{Statistical analysis}

All the statistical data were analyzed using STATA 14.2 software. Pooled odds ratios (ORs) with 95\% confidence intervals (CIs) were calculated to assess the relationship between high FTX expression and clinicopathological characteristics that included sex (male vs. female), tumor size (larger size vs. smaller size), differentiation (low vs. high-moderate), lymph node metastasis (yes vs. no), distant metastasis (yes vs. no), and TNM/clinical stage (III+IV vs. I+II). HRs with $95 \%$ CIs were calculated to assess the correlation between the overexpression of FTX and survival outcome. HRs and 95\% CIs analyzed by Cox regression analysis were extracted directly from publications. If HRs and 95\% CIs analyzed by the Kaplan-Meier method could not be obtained, the software Engauge Digitizer and published methods [18] were used to extract the survival data from a Kaplan-Meier curve in related articles. Heterogeneity was assessed by the $I^{2}$ test and $Q$ test, and the random effects model was used if the $I^{2}>50 \%$, otherwise, a fixed effects 


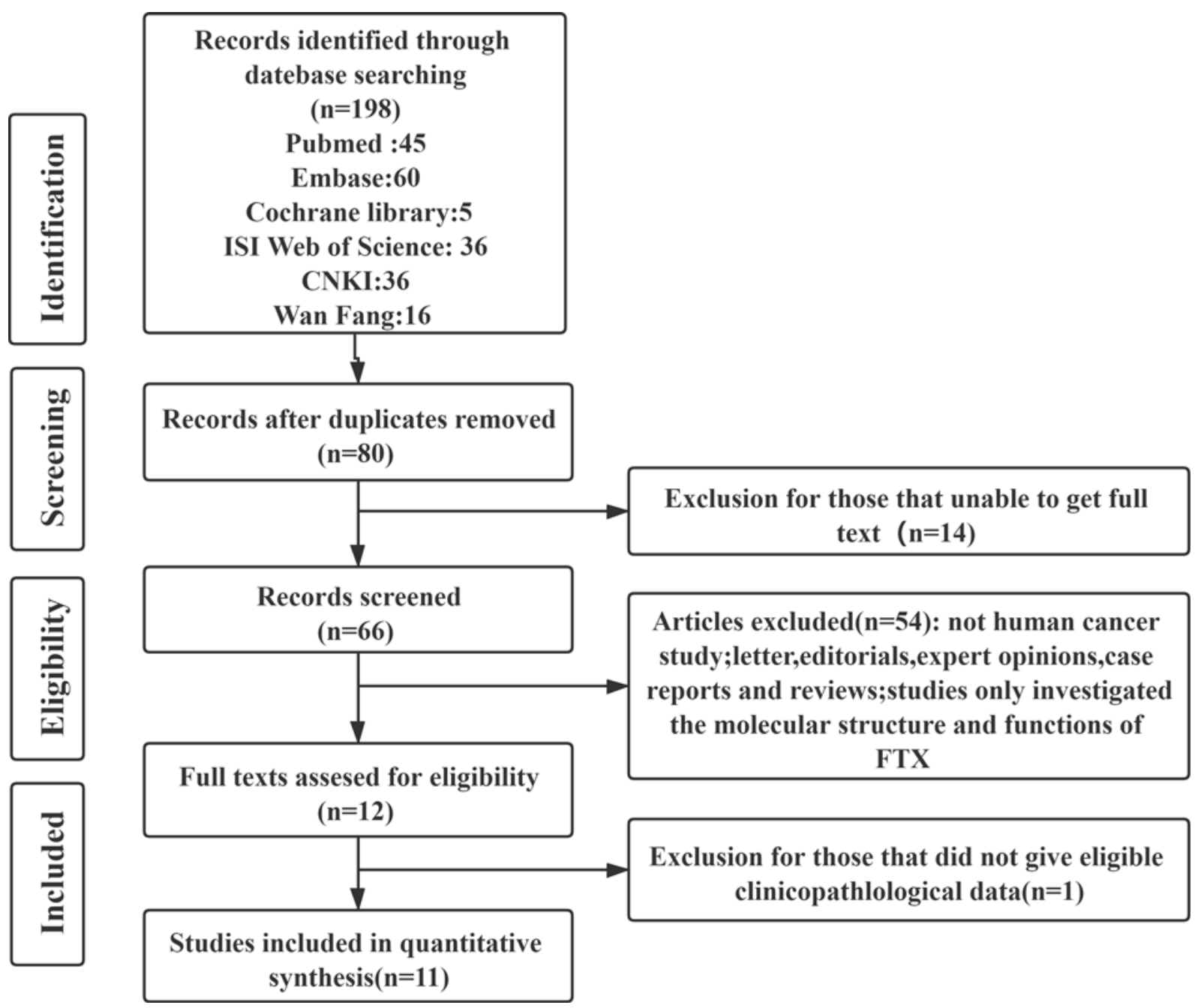

Figure 1. Flow diagram of study

model was used. The potential publication bias was assessed by Begg's funnel analysis. $P<0.05$ was considered to be statistically significant. Sensitivity analysis was used to calculate the source of heterogeneity and stability of results.

\section{Results}

\section{Study identification and characteristics}

As shown by the search flow diagram (Figure 1), a total of 11 studies $[9-13,15,19-23]$ with 1210 patients were enrolled in this meta-analysis satisfied the inclusion criteria, including 3 studies on CRC, 3 studies on HCC, 2 studies on GC, and 1 study each on RCC, OSC, and glioma. The included studies were published from 2015 to 2020, and sample sizes ranged from 30 to 187 patients. All of included studies were from China. Nine studies were published in English and two in Chinese. All of included studies received scores $\geq 6$ in methodological assessments, which meant they had high quality. The clinicopathological characteristics and survival information obtained from these studies are summarized in Tables 1 and 2.

\section{FTX expression and clinicopathological characteristics}

The pooled ORs with 95\% CI are shown in Table 3 . The results revealed that elevated FTX expression was significantly associated with lymph node metastasis (yes vs. no: $\mathrm{OR}=2.47,95 \% \mathrm{CI}[1.45,4.22], P=0.001$, Figure $2 \mathrm{~A}$ ) in seven studies (three studies on CRC, two studies on GC, and one study each on HCC and RCC) $[9,11,12,15,20-22]$, distant metastasis (yes vs. no: $\mathrm{OR}=3.87,95 \% \mathrm{CI}[2.38,6.30], P<0.001$, Figure $2 \mathrm{~B}$ ) in five studies (two studies on $\mathrm{CRC}$ and one study each on GC, HCC, and OSC) $[9,10,15,21,22]$, bigger tumor size (larger vs. smaller: OR $=2.10,95 \%$ CI 
Table 1 Characteristics of studies included in the meta-analysis

Study

Cancer Detection Sample

Survival

infor- NOS

Year Countrytype method size LncRNA FTX expression

mation score

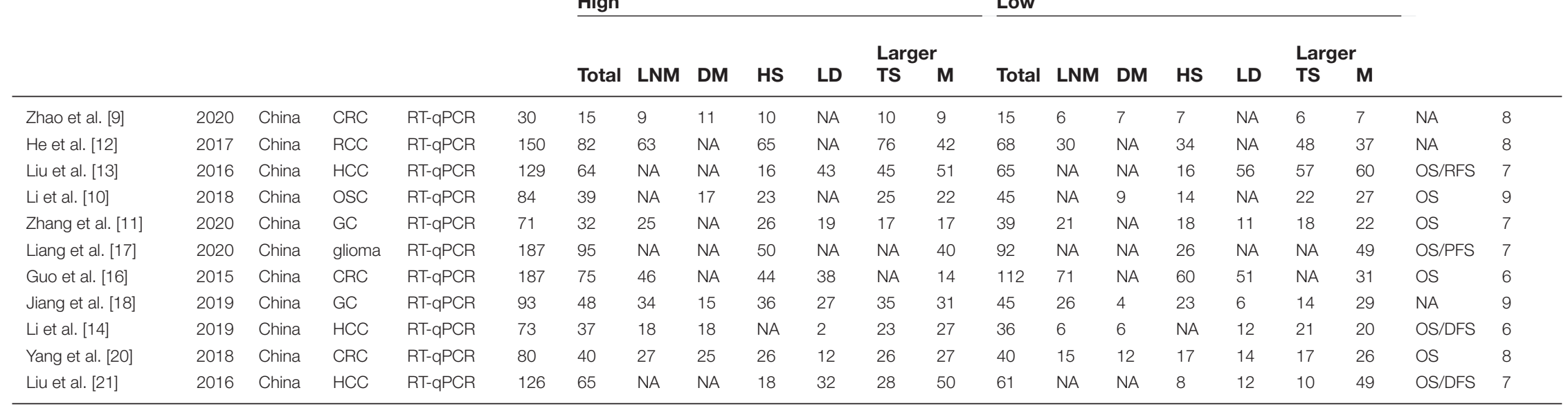

Abbreviations: CRC, colorectal cancer; DFS, disease-free survival; DM, distant metastasis; GC, gastric cancer; HCC, hepatocellular carcinoma; HS, high TNM/clinical stage (III/IV); Larger

TS, the larger tumor size group (diameter $\geq 3 \mathrm{~cm}$ ); LD, low differentiation; LNM, Iymph node metastasis; M, man; OS, overall survival; NA, not available; NOS, Newcastle-Ottawa scale; OSC, osteosarcoma; PFS, progression-free survival; RCC, renal cell carcinoma; RFS, recurrence-free survival; RT-qPCR, reverse transcription quantitative real-time polymerase chain reaction. 
Table 2 Survival data of studies included in the meta-analysis

\begin{tabular}{|c|c|c|c|c|c|c|c|c|c|}
\hline \multirow[t]{3}{*}{ Study } & \multirow[t]{3}{*}{ Year } & \multirow[t]{3}{*}{$\begin{array}{l}\text { Cancer } \\
\text { type }\end{array}$} & \multirow[t]{3}{*}{$\begin{array}{l}\text { Detection } \\
\text { method }\end{array}$} & \multirow[t]{3}{*}{$\begin{array}{l}\text { Sample } \\
\text { size }\end{array}$} & \multicolumn{4}{|c|}{ Survival information } & \multirow[t]{3}{*}{ HR statistic } \\
\hline & & & & & \multicolumn{2}{|c|}{ Overall survival (OS) } & \multicolumn{2}{|l|}{ Other survival } & \\
\hline & & & & & $\begin{array}{l}\text { Univariate } \\
\text { analysis }\end{array}$ & $\begin{array}{l}\text { Multivari- } \\
\text { ate }\end{array}$ & $\begin{array}{l}\text { Univariate } \\
\text { analysis }\end{array}$ & $\begin{array}{l}\text { Multivari- } \\
\text { ate }\end{array}$ & \\
\hline Liu et al [13] & 2016 & $\mathrm{HCC}$ & RT-qPCR & 129 & $0.59(0.34-1.03)(E)$ & NA & $0.51(0.30-0.89)(E)^{1}$ & NA & Survival curve \\
\hline Li et al [10] & 2018 & OSC & RT-qPCR & 84 & $2.636(1.458-4.767)$ & $\begin{array}{l}2.162 \\
(1.158-4.037)\end{array}$ & NA & NA & Data in paper \\
\hline Zhang et al [11] & 2020 & GC & RT-qPCR & 71 & $2.07(1.33-3.24)(E)$ & NA & NA & NA & Survival curve \\
\hline Liang et al [17] & 2020 & glioma & RT-qPCR & 187 & $1.36(0.90-2.07)(E)$ & $\begin{array}{l}5.358 \\
(1.775-8.569)\end{array}$ & $1.69(1.15-2.49)(E)^{2}$ & $\begin{array}{l}6.323 \\
(2.213-9.934)^{2}\end{array}$ & Both of two \\
\hline Guo et al [16] & 2015 & $\mathrm{CRC}$ & RT-qPCR & 187 & $151(0.98-2.32)(E)$ & $\begin{array}{l}2.371 \\
(1.42-2.739)\end{array}$ & NA & NA & Both of two \\
\hline Li et al [14] & 2019 & $\mathrm{HCC}$ & RT-qPCR & 73 & $\begin{array}{l}12.456 \\
(1.607-96.543)\end{array}$ & $\begin{array}{l}10.376 \\
(1.284-83.836)\end{array}$ & $\begin{array}{l}7.484 \\
(3.398-16.486)^{3}\end{array}$ & $\begin{array}{l}6.411 \\
(2.859-14.375)^{3}\end{array}$ & $\begin{array}{l}\text { Data in paper } \\
)^{3}\end{array}$ \\
\hline Yang et al [20] & 2018 & $\mathrm{CRC}$ & RT-qPCR & 80 & $1.32(0.80-2.17)(E)$ & NA & NA & NA & Survival curve \\
\hline Liu et al [21] & 2016 & $\mathrm{HCC}$ & RT-qPCR & 126 & $1.92(1.23-2.92)(E)$ & NA & $2.08(1.32-3.27)(E)^{3}$ & NA & Survival curve \\
\hline \multicolumn{10}{|c|}{$\begin{array}{l}{ }^{1} \text { Recurrence-free survival. } \\
2 \text { Progression-free survival. } \\
{ }^{3} \text { Disease-free survival. } \\
\text { Abbreviations: CRC, colorectal cancer; E, survival data were obtained by using software Engauge Digitizer; GC, gastric cancer; HCC, hepatocellular } \\
\text { carcinoma; HR, hazard ratio; NA, not available; OSC, osteosarcoma; RCC, renal cell carcinoma; RT-qPCR, reverse transcription quantitative real-time } \\
\text { polymerase chain reaction. }\end{array}$} \\
\hline
\end{tabular}

Table 3 LncRNA FTX clinicopathological features for cancers

\begin{tabular}{|c|c|c|c|c|c|c|c|}
\hline $\begin{array}{l}\text { Clinicopatholog } \\
\text { features }\end{array}$ & $\begin{array}{l}\text { cal } \\
\text { No. of studies }\end{array}$ & No. of patients & $\begin{array}{l}\text { Pooled OR } \\
(95 \% \mathrm{Cl})\end{array}$ & $P$ Het & $I^{2}(\%)$ & $P$ value & Model used \\
\hline Gender & 11 & 1210 & $0.87(0.68-1.12)$ & 0.555 & 0 & 0.271 & Fixed \\
\hline $\begin{array}{l}\text { Lymph node } \\
\text { metastasis }\end{array}$ & 7 & 684 & $2.47(1.45-4.22)$ & 0.002 & 59.5 & 0.001 & Random \\
\hline Distant metastasis & 5 & 360 & $3.87(2.38-6.30)$ & 0.973 & 0 & $<0.001$ & Fixed \\
\hline Tumor size & 9 & 836 & $2.10(1.15-3.85)$ & 0 & 72.5 & 0.016 & Random \\
\hline Differentiation & 7 & 759 & $1.33(0.53-3.33)$ & 0 & 86.2 & 0.546 & Random \\
\hline TNM/clinical stage & 10 & 1137 & $2.38(1.85-3.06)$ & 0.248 & 21.2 & $<0.001$ & Fixed \\
\hline
\end{tabular}

Abbreviations: Cl, confidence interval; Fixed, fixed effects model; OR, odds ratio; Random, random effects model.

$[1.15,3.85], P=0.016$, Figure 2C) in nine studies (two studies each on CRC and GC, three studies on HCC, and one study each on RCC and OSC) [9-13,15,21-23], and subsequently on TNM/clinical stage (III+IV vs. I+II : OR $=2.38$, $95 \%$ CI $[1.85,3.06], P<0.001$, Figure 2D in ten studies (three studies on CRC, two studies each on GC and HCC, one study each on RCC, OSC, glioma) [9-13,19-23]. The overexpression of FTX was not associated with sex (male vs. female: $\mathrm{OR}=0.87,95 \% \mathrm{CI}[0.68,1.12], P=0.271$, Figure $2 \mathrm{E}$ ) in all the 11 aforementioned studies and differentiation (low vs. high and moderate: $\mathrm{OR}=1.33,95 \% \mathrm{CI}[0.53,3.33], P=0.546$, Figure $2 \mathrm{~F}$ ) in seven studies (two studies on CRC, three studies on GC, and two studies on HCC) $[11,13,15,20-23]$. The findings suggest that high expression of FTX is associated with clinicopathological parameters of the aforementioned cancers.

\section{FTX expression and survival outcomes}

A meta-analysis was conducted using the data from survival analysis using the Kaplan-Meier method in eight studies (three studies on HCC, two studies on CRC, and one study each on GC, OSC, and glioma) $[10,11,13,15,19,20,22,23]$. The pooled HRs for OS was extracted from eight heterogeneous studies $\left(I^{2}=68.3 \%, P=0.002\right)$ comprising 937 patients, and the results showed a significant correlation between high FTX expression and a shorter OS (high vs. low: $\mathrm{HR}=1.58,95 \% \mathrm{CI}[1.13,2.20], P=0.007$, Figure $3 \mathrm{~A})$. In addition, the two heterogeneous studies $[15,23]\left(I^{2}=86.8 \%\right.$, 
(A)

Study

ID

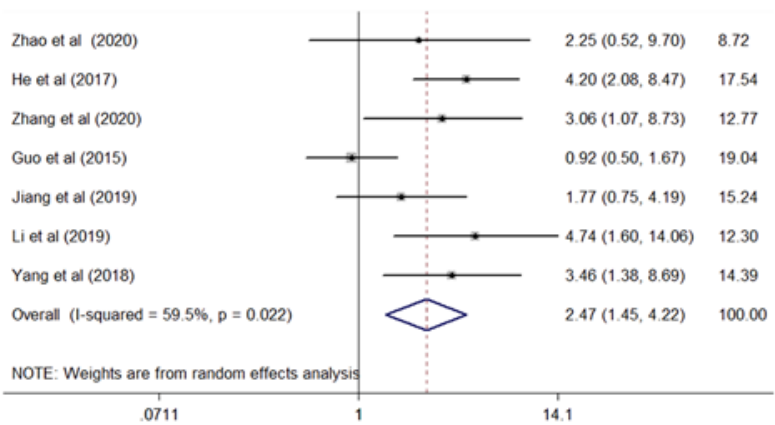

(C)

Study

ID

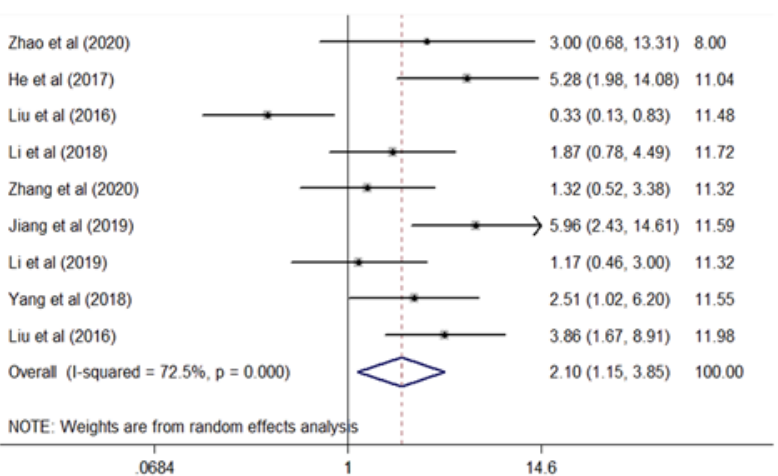

(E)

Study

ID

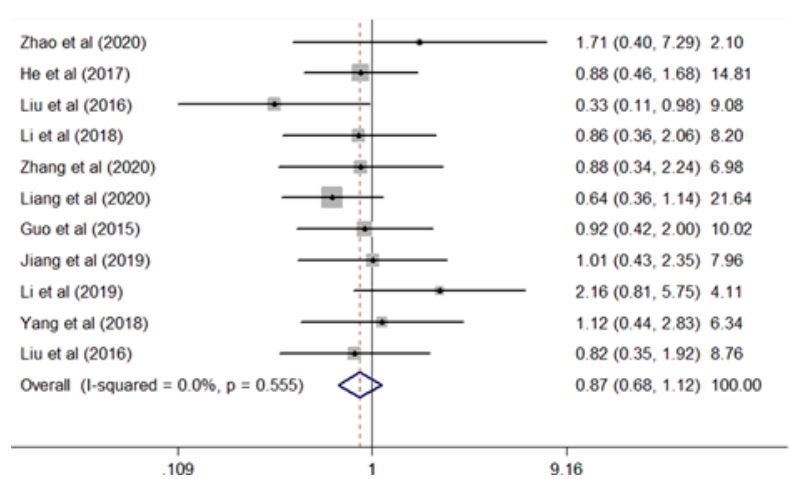

(B)

Study

ID

OR $(95 \% \mathrm{Cl}) \quad$ Weight

Zhao et al (2020)

Li et al (2018)

Jiang et al (2019)

Li et al (2019)

Yang et al (2018)

Overal (l-squared $=0.0 \%, p=0.973$ )

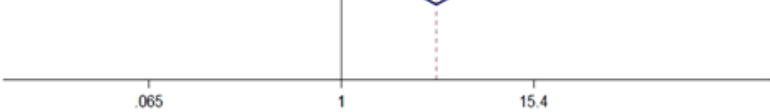

(D)

Study

ID

OR $(95 \% \mathrm{Cl}) \quad$ Weight

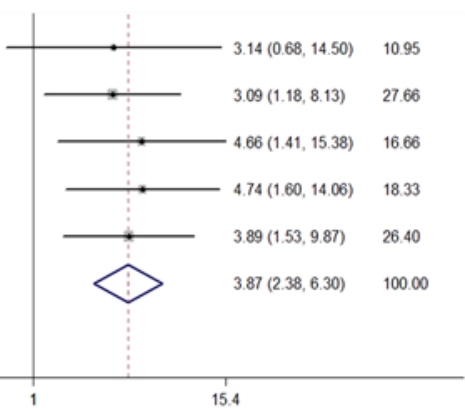

al (2020)

He et al (2017)

Liv et al (2016)

Li et al (2018)

Zhang et al (2020)

Liang et al (2020)

Guo et al (2015)

Jiang et al (2019)

Yang et al (2018)

Liv et al (2016)

Overall (1-squared $=21.2 \%, p=0.248$ )

\begin{tabular}{c|ccc} 
& & & \\
\hline 1 & & & \\
\hline 1 & 1566
\end{tabular}

(F)

Study

$\%$

ID

OR $(95 \% \mathrm{Cl}) \quad$ Weight

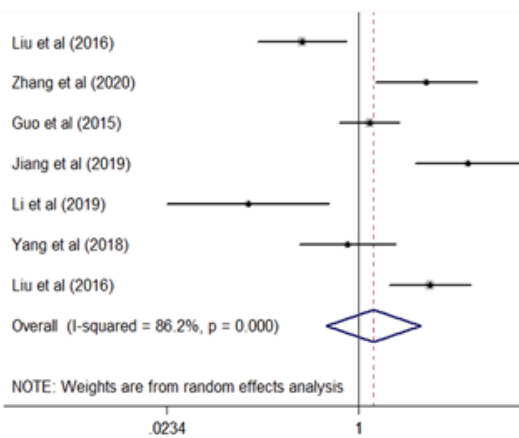

$0.33(0.14,0.79) \quad 14.78$

$3.72(1.38,10.03) \quad 14.24$

$1.23(0.68,2.21) \quad 15.96$

$8.36(2.98,23.44) \quad 14.05$

$0.11(0.02,0.56) \quad 11.33$

$0.80(0.31,2.03) \quad 14.50$

$3.96(1.78,8.78) \quad 15.13$

$1.33(0.53,3.33) \quad 100.00$

Figure 2. Forest plot of studies evaluating the relationship between FTX expression and clinicopathological features

(A) Lymph node metastasis, (B) distant metastasis, (C) tumor size, (D) TNM/clinical stage, (E) gender, (F) differentiation.

$P=0.006$ ) with 199 patients with HCC were included, and the pooled results indicated that high FTX expression was related to a shorter DFS (high vs. low: $\mathrm{HR}=3.78,95 \% \mathrm{CI}=[1.08,13.22], P=0.037$, Figure $3 \mathrm{~B}$ ).

Another meta-analysis was conducted using data from the survival analysis in a multivariate Cox regression model that included four studies (one study each on CRC, HCC, OSC, and glioma) and a total of 531 patients [10,15,19,20]. 
(A)

Study

ID

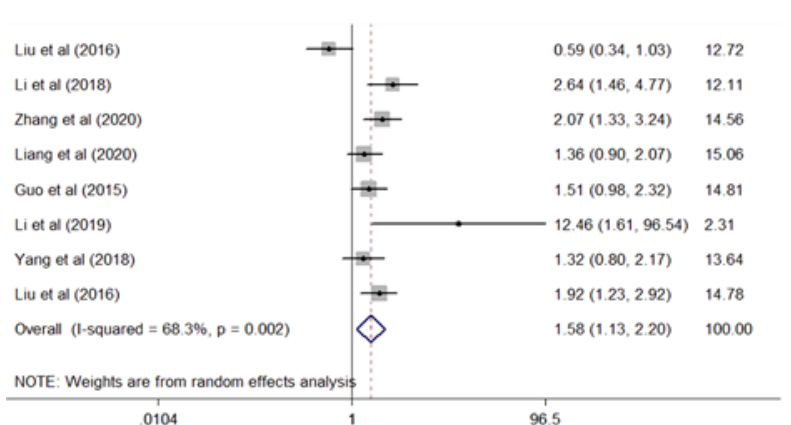

(C)

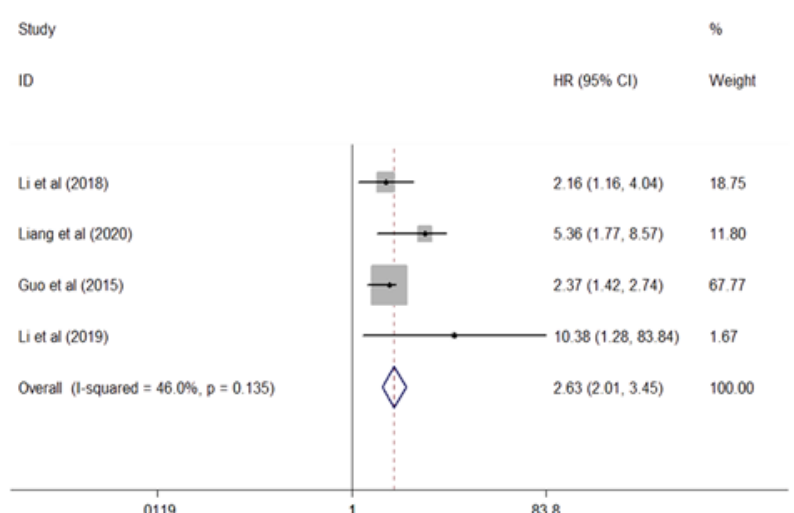

(B)

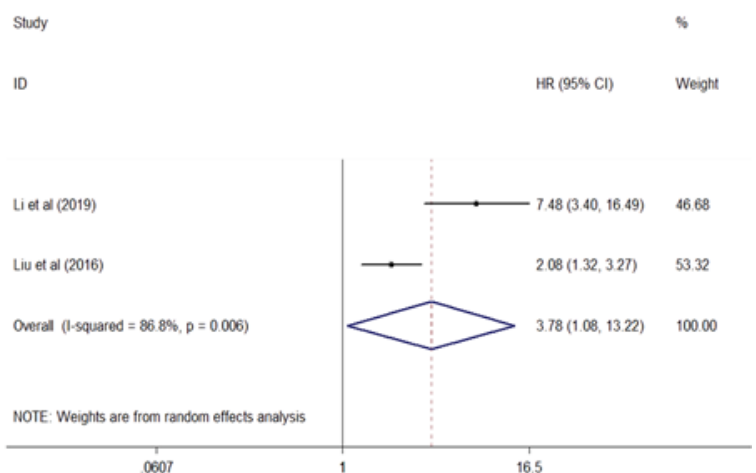

Figure 3. Forest plot of studies evaluating the relationship between FTX expression and the survival rate (A) Overall survival (OS) rate, (B) disease-free survival (DFS) rate, (C) independent predictive factor for OS.

The role of FTX as an independent predictive factor for OS in patients with cancer was assessed. The pooled results showed that higher FTX expression was an independent prognostic factor for a shorter OS of patients with the aforementioned four cancers ( $\mathrm{HR}=2.63,95 \% \mathrm{CI}[2.01,3.45], P<0.001$, Figure $3 \mathrm{C}$ ).

\section{Sensitivity analysis}

We performed sensitivity analyses to evaluate the robustness of the prognostic model. The results (Supplementary Tables S1 and S2) were not significantly altered when any individual study was removed, demonstrating that the results were reliable.

\section{Publication bias}

Begg's funnel analysis was conducted to evaluate the publication bias. As shown in Figure 4, there was no publication bias for sex $(P=0.276)$, lymph node metastasis $(P=0.764)$, distant metastasis $(P=1.0)$, tumor size $(P=0.754)$, differentiation $(P=1.0)$, TNM/clinical stage $(P=0.858)$, OS $(P=0.174)$ or DFS $(P=1.0)$, and the model was an independent factor for OS $(P=0.308)$.

\section{Discussion}

In the 1980s, the first regulatory non-coding RNA (ncRNA) was identified in bacteria, and subsequently, a large number of ncRNAs have been found in most eukaryotic organisms [24]. LncRNAs belong to this class of ncRNAs and were initially dismissed as being merely transcriptional 'noise' [25]. However, emerging evidence has revealed that lncRNAs are associated with the development of cancer by regulating various cellular functions, including proliferation, migration, and DNA stability [26]. Because of the tissue-specific characteristics of lncRNAs, they may become the next generation of biomarkers or targets for human cancer [27]. 


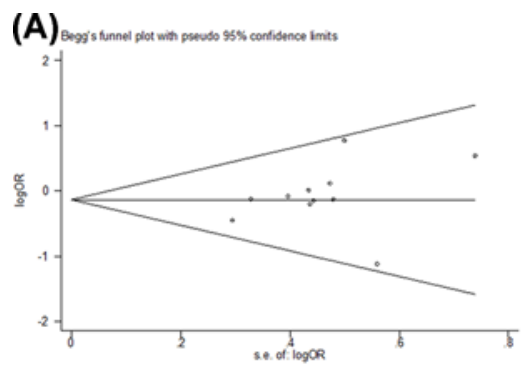

(D)

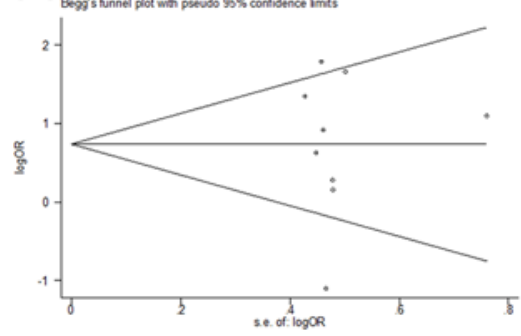

(G)

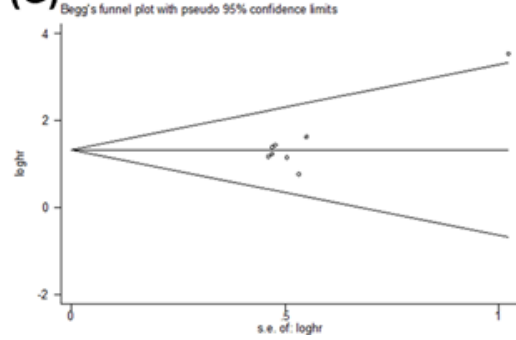

(B)

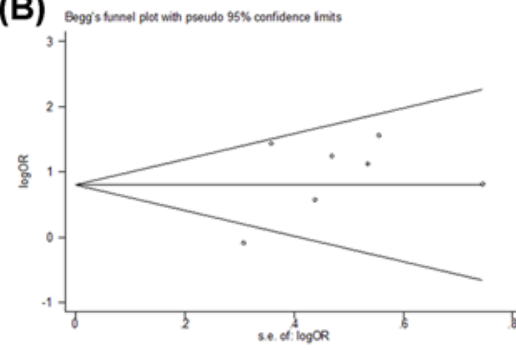

(E)

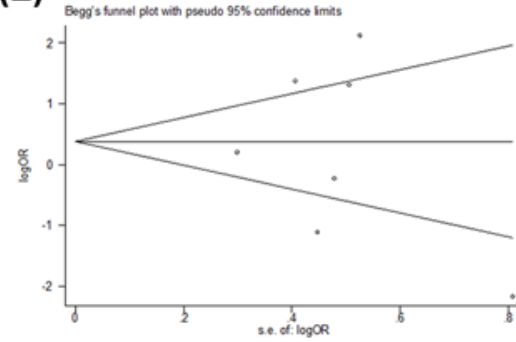

(H)

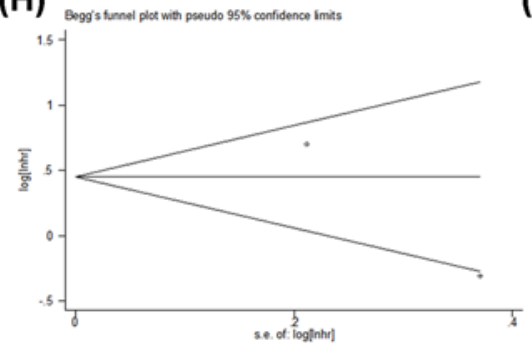

(C)

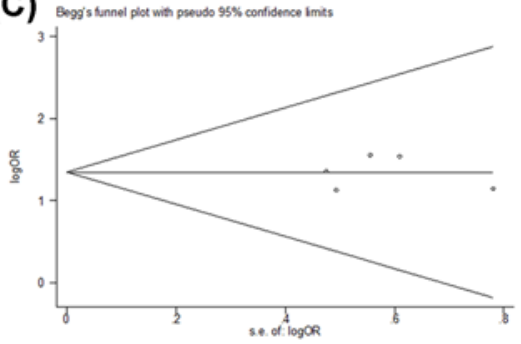

(F)

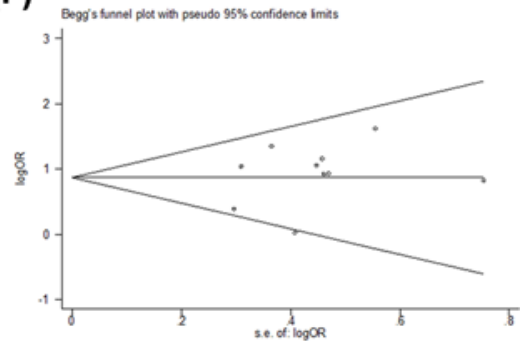

(I)

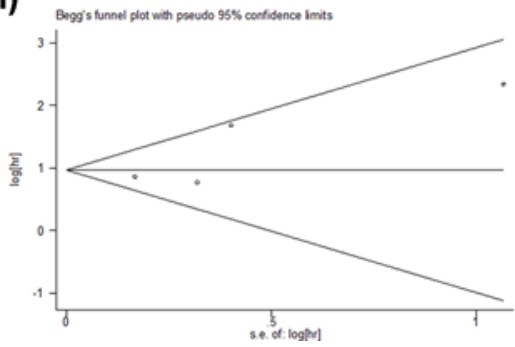

Figure 4. Begg's publication bias plots for FTX related studies

(A) Gender, (B) lymph node metastasis, (C) distant metastasis, (D) tumor size, (E) differentiation, (F) TNM/clinical stage, (G) overall survival (OS) rate, (H) disease-free survival (DFS) rate, and (I) independent predictive factor for OS.

FTX is a member of the lncRNA family. In recent years, changes in FTX expression in different types of cancers and the influence of FTX expression in cancer have attracted much attention. Nonetheless, the effects of FTX expression on the development and prognosis of cancer are still controversial. Therefore, we combined all published studies investigating this issue to conduct this meta-analysis.

A total of 11 eligible studies comprising 1210 cancer patients, reporting six common cancer types, were included. Our results showed that high FTX expression was significantly associated with several clinicopathological characteristics, including lymph node metastasis in patients with CRC, GC, HCC, and RCC; distant metastasis in patients with CRC, GC, HCC, and OSC; bigger tumor size in patients with CRC, GC, HCC, RCC, and OSC; and later TNM/clinical stage in patients with CRC, GC, HCC, OSC, and glioma. However, there were no significant differences between the high and low FTX expression groups with respect to sex and tumor differentiation. Conversely, a significant association between high FTX expression and a shorter OS in patients with CRC, GC, HCC, OSC, and glioma, or a shorter DFS in patients with HCC was observed. Further meta-analysis showed that high FTX expression could be an independent predictive marker for shorter OS in patients with CRC, HCC, OSC, and glioma. Taken together, these results suggested that FTX may be a candidate oncogene, and the overexpression of FTX was associated with a poor prognosis in common solid malignant tumors, such as CRC, HCC, OSC, and glioma.

Based on evidence from previous studies, cancer promotion by FTX may involve the following mechanisms: FTX significantly promotes the proliferation and invasion of glioma cells by negatively regulating miR-342-3p [16]; FTX exerts its oncogenic role in OSC via up-regulating the expression of TXNRD1 through sponging miR-320a [28]; FTX promotes proliferation and invasion of gastric cancer via the miR-144/ZFX axis [29]; FTX functions as an oncogene to contribute to CRC progression by regulating the miR-192-5p/EIF5A2 axis [9].

Our results were similar to those of previous reports. For example, in the meta-analysis investigating the lncRNA GHET-1, GHET-1 expression was closely correlated with tumor size, lymph node metastasis, distant metastasis and 
TNM stage, and increased GHET-1 expression may be a potential prognostic biomarker in human cancers including HCC, GC,OSC, breast cancer, baldder cancer, cervical cancer, NSCLC, and esophageal cancer (ESCC) [30]. Zhu et al. [31] conducted a meta-analysis which demonstrated that elevated IncRNA XIST expression predicted poor OS, poor DFS, larger tumor size, increased distant metastasis and advanced tumor stage, suggesting that high lncRNA XIST expression may serve as a novel biomarker for poor prognosis and metastasis in cancers (CRC, GC, HCC, ESCC, pancreatic cancer [PC], nasopharyngeal carcinoma [NPC], and glioma).

Nevertheless, several limitations existed in this meta-analysis. The main limitation of the present study was the small number of included studies and patients (11 studies and 1210 patients), thus further subgroup analysis could not be performed stratifying patients by age group and tumor site. Subgroup analysis was not carried out for different types of tumors to reduce the heterogeneity caused by different types of tumors. Second, all of the studies were from China, so the results may only be applicable to the Chinese populations. Large-scale and well-designed studies are still needed to verify the clinical value of FTX in different ethnicities. Third, some studies did not provide HRs directly, and an available software was used to calculate the HRs values, which may have introduced errors. Fourth, the included studies had different criteria for the classification of FTX expression, which may have affected the heterogeneity of the meta-analysis.

\section{Conclusions}

FTX may be a potential oncogene, and its high expression be associated with a poor prognosis in patients with CRC, HCC, OSC, and glioma. Additional high-quality studies involving large numbers of patients are needed to confirm the findings.

\section{Data Availability}

The data used to support the findings of this study are included within the article. The primary data used to support the findings of this study are available from the corresponding author upon request.

\section{Competing Interests}

The authors declare that there are no competing interests associated with the manuscript.

\section{Funding}

The authors declare that there are no sources of funding to be acknowledged.

\section{Author Contribution}

Idea and design: T.S.H. and C.W.W. Data collection: C.W.W. and L.Y.T. Data analysis: Z.C.X. and G.L.L.Z. Manuscript writing: C.W.W. Manuscript revision: T.S.H. and C.W.W. All authors read and approved the version of the manuscript to be published. All authors take responsibility for appropriate content.

\section{Acknowledgements}

We would like to thank the researchers and study participants for their contributions.

\section{Abbreviations}

$\mathrm{Cl}$, confidence interval; $\mathrm{CRC}$, colorectal cancer; DFS, disease-free survival; DM, distant metastasis; ESCC, esophageal cancer; GC, gastric cancer; HCC, hepatocellular carcinoma; HR, hazard ratio; NPC, nasopharyngeal carcinoma; OR, odds ratio; OS, overall survival; OSC, osteosarcoma; RCC, renal cell carcinoma; RT-qPCR, reverse transcription quantitative real-time polymerase chain reaction.

\section{References}

1 Bray, F., Ferlay, J., Soerjomataram, I., Siegel, R.L., Torre, L.A. and Jemal, A. (2018) Global cancer statistics 2018: GLOBOCAN estimates of incidence and mortality worldwide for 36 cancers in 185 countries. CA Cancer J. Clin. 68, 394-424, https://doi.org/10.3322/caac.21492

2 Wu, C., Li, M., Meng, H., Liu, Y., Niu, W., Zhou, Y. et al. (2019) Analysis of status and countermeasures of cancer incidence and mortality in China. Sci. China Life Sci. 62, 640-647, https://doi.org/10.1007/s11427-018-9461-5

3 Tsai, M.C., Spitale, R.C. and Chang, H.Y. (2011) Long intergenic noncoding RNAs: new links in cancer progression. Cancer Res. 71, 3-7, https://doi.org/10.1158/0008-5472.CAN-10-2483

4 Cui, X., Jing, X., Long, C., Tian, J. and Zhu, J. (2017) Long noncoding RNA MEG3, a potential novel biomarker to predict the clinical outcome of cancer patients: a meta-analysis. Oncotarget 8, 19049-19056, https://doi.org/10.18632/oncotarget.14987 
5 Lin, Y., Sheng, Y., Chen, J., Hu, C., Zhou, Z. and Yuan, C. (2020) The Function of LncRNA FTX in Several Common Cancers. Curr. Pharm. Des., pii:CPD-EPUB-111038, https://doi.org/10.2174/1381612826666201029164036

6 Renganathan, A. and Felley-Bosco, E. (2017) Long Noncoding RNAs in Cancer and Therapeutic Potential. Adv. Exp. Med. Biol. 1008, 199-222, https://doi.org/10.1007/978-981-10-5203-37

7 Bhan, A., Soleimani, M. and Mandal, S.S. (2017) Long Noncoding RNA and Cancer: A New Paradigm. Cancer Res. 77, 3965-3981, https://doi.org/10.1158/0008-5472.CAN-16-2634

8 Chureau, C., Chantalat, S., Romito, A., Galvani, A., Duret, L., Avner, P. et al. (2011) Ftx is a non-coding RNA which affects Xist expression and chromatin structure within the X-inactivation center region. Hum. Mol. Genet. 20, 705-718, https://doi.org/10.1093/hmg/ddq516

9 Zhao, K., Ye, Z., Li, Y., Li, C., Yang, X., Chen, Q. et al. (2020) LncRNA FTX Contributes to the Progression of Colorectal Cancer Through Regulating miR-192-5p/EIF5A2 Axis. OncoTargets Ther. 13, 2677-2688, https://doi.org/10.2147/0TT.S241011

10 Li, B., Ren, P. and Wang, Z. (2018) Long non-coding RNA Ftx promotes osteosarcoma progression via the epithelial to mesenchymal transition mechanism and is associated with poor prognosis in patients with osteosarcoma. Int. J. Clin. Exp. Pathol. 11, 4503-4511

11 Zhang, F., Wang, X.S., Tang, B., Li, P.A., Wen, Y. and Yu, P.W. (2020) Long non-coding RNA FTX promotes gastric cancer progression by targeting miR-215. Eur. Rev. Med. Pharmacol. Sci. 24, 3037-3048

12 He, X., Sun, F., Guo, F., Wang, K., Gao, Y., Feng, Y. et al. (2017) Knockdown of Long Noncoding RNA FTX Inhibits Proliferation, Migration, and Invasion in Renal Cell Carcinoma Cells. Oncol. Res. 25, 157-166, https://doi.org/10.3727/096504016X14719078133203

13 Liu, F., Yuan, J.H., Huang, J.F., Yang, F., Wang, T.T., Ma, J.Z. et al. (2016) Long noncoding RNA FTX inhibits hepatocellular carcinoma proliferation and metastasis by binding MCM2 and miR-374a. Oncogene 35, 5422-5434, https://doi.org/10.1038/onc.2016.80

14 Jin, S., He, J., Zhou, Y., Wu, D., Li, J. and Gao, W. (2020) LncRNA FTX activates FOXA2 expression to inhibit non-small-cell lung cancer proliferation and metastasis. J. Cell. Mol. Med. 24, 4839-4849, https://doi.org/10.1111/jcmm.15163

$15 \mathrm{Li}, \mathrm{X}$. (2019) The Role and mechanism of long non-coding RNA Ftx in the aerobic glycolysis and progression in hepatocellular carcinoma, Shandong University, (Chinese)

16 Zhang, W., Bi, Y., Li, J., Peng, F., Li, H., Li, C. et al. (2017) Long noncoding RNA FTX is upregulated in gliomas and promotes proliferation and invasion of glioma cells by negatively regulating miR-342-3p. Lab. Invest. 97, 447-457, https://doi.org/10.1038/labinvest.2016.152

17 Stang, A. (2010) Critical evaluation of the Newcastle-Ottawa scale for the assessment of the quality of nonrandomized studies in meta-analyses. Eur. J. Epidemiol. 25, 603-605, https://doi.org/10.1007/s10654-010-9491-z

18 Tierney, J.F., Stewart, L.A., Ghersi, D., Burdett, S. and Sydes, M.R. (2007) Practical methods for incorporating summary time-to-event data into meta-analysis. Trials 8, 16, https://doi.org/10.1186/1745-6215-8-16

19 Liang, Y. and Lu, H. (2020) Long noncoding RNA FTX is associated with prognosis of glioma patients. J. Gene Med. 22, e3237, https://doi.org/10.1002/jgm.3237

20 Guo, X.B., Hua, Z., Li, C., Peng, L.P., Wang, J.S., Wang, B. et al. (2015) Biological significance of long non-coding RNA FTX expression in human colorectal cancer. Int. J. Clin. Exp. Med. 8, 15591-15600

21 Yang, Y., Zhang, J., Chen, X., Xu, X., Cao, G., Li, H. et al. (2018) LncRNA FTX sponges miR-215 and inhibits phosphorylation of vimentin for promoting colorectal cancer progression. Gene Ther. 25, 321-330, https://doi.org/10.1038/s41434-018-0026-7

22 Yang, Y., Zhang, J., Chen, X., Xu, X., Cao, G., Li, H. et al. (2018) LncRNA FTX sponges miR-215 and inhibits phosphorylation of vimentin for promoting colorectal cancer progression. Gene Ther. 25, 321-330, https://doi.org/10.1038/s41434-018-0026-7

23 Liu, Z., Dou, C., Yao, B., Xu, M., Ding, L., Wang, Y. et al. (2016) Ftx non coding RNA-derived miR-545 promotes cell proliferation by targeting RIG-I in hepatocellular carcinoma. Oncotarget 7, 25350-25365, https://doi.org/10.18632/oncotarget.8129

24 Jarroux, J., Morillon, A. and Pinskaya, M. (2017) History, Discovery, and Classification of IncRNAs. Adv. Exp. Med. Biol. 1008, 1-46, https://doi.org/10.1007/978-981-10-5203-31

25 Ponting, C.P., Oliver, P.L. and Reik, W. (2009) Evolution and functions of long noncoding RNAs. Cell 136, 629-641, https://doi.org/10.1016/j.cell.2009.02.006

26 Rathinasamy, B. and Velmurugan, B.K. (2018) Role of IncRNAs in the cancer development and progression and their regulation by various phytochemicals. Biomed. Pharmacother. 102, 242-248, https://doi.org/10.1016/j.biopha.2018.03.077

$27 \mathrm{Wu}$, T. and Du, Y. (2017) LncRNAs: From Basic Research to Medical Application. Int. J. Biol. Sci. 13, 295-307, https://doi.org/10.7150/ijbs.16968

28 Huang, S., Zhu, X., Ke, Y., Xiao, D., Liang, C., Chen, J. et al. (2020) LncRNA FTX inhibition restrains osteosarcoma proliferation and migration via modulating miR-320a/TXNRD1. Cancer Biol. Ther. 21, 379-387, https://doi.org/10.1080/15384047.2019.1702405

29 Li, H., Yao, G., Zhai, J., Hu, D. and Fan, Y. (2019) LncRNA FTX Promotes Proliferation and Invasion of Gastric Cancer via miR-144/ZFX Axis. OncoTargets Ther. 12, 11701-11713, https://doi.org/10.2147/0TT.S220998

30 Wang, D., Zhang, H., Fang, X., Zhang, X. and Liu, H. (2020) Prognostic value of long non-coding RNA GHET1 in cancers: a systematic review and meta-analysis. Cancer Cell Int. 20, 109, https://doi.org/10.1186/s12935-020-01189-9

31 Zhu, J., Kong, F., Xing, L., Jin, Z. and Li, Z. (2018) Prognostic and clinicopathological value of long noncoding RNA XIST in cancer. Clin. Chim. Acta 479, 43-47, https://doi.org/10.1016/j.cca.2018.01.005 